\title{
A TAUBERIAN THEOREM FOR HAUSDORFF METHODS
}

\author{
BRIAN KUTTNER AND MANGALAM R. PARAMESWARAN
}

(Communicated by R. Daniel Mauldin)

Dedicated to the memory of Professor V. Ganapathy Iyer

\begin{abstract}
Let $H=(H, \chi)$ be a regular Hausdorff summability method defined by the function $\chi \in \mathrm{BV}[0,1]$. It is shown that if $\chi$ is absolutely continuous on $[0,1]$, then the methods $H$ and $V \cdot H$ are equivalent for bounded sequences, where $V$ belongs to a certain class of summability methods which includes the Cesàro methods $C_{\alpha}(\alpha>0)$, the Abel method $A$, and the methods $A \cdot C_{\alpha}(\alpha>-1)$.
\end{abstract}

1. Introduction. The Hausdorff method $H=(H, \chi)$, where $\chi \in \mathrm{BV}[0,1]$ transforms sequences $s=\left\{s_{k}\right\}$ into sequences $H s=t=\left\{t_{k}\right\}(k \geqslant 0)$, where

$$
t_{n}=\int_{0}^{1} \sum_{k=0}^{n}\left(\begin{array}{l}
n \\
k
\end{array}\right) t^{k}(1-t)^{n-k} s_{k} d \chi(t) \quad(n \geqslant 0) .
$$

If $V_{1}, V_{2}$ are summability methods, we write $V_{1} \sim V_{2}$ for bounded sequences if $V_{1}$ and $V_{2}$ are equivalent for bounded sequences, i.e. a bounded sequence is $V_{1}$-summable if and only if it is $V_{2}$-summable (not necessarily to the same limit). (For detailed properties of the Abel, Cesàro, and Hausdorff methods in general, see [2 or 8].) Stam [7] showed recently that every $C_{1}$-summable bounded sequence is summable by $H=(H, \chi)$ if $\chi(t)$ is absolutely continuous on [0,1]. We now prove a stronger result, by making use of a Modified Reduction Principle-a concept introduced by the second author as a valuable tool in Tauberian theory (see e.g. [3, 4, and 5]).

\section{Theorem.}

THEOREM. Let $H=(H, \chi)$ be a Hausdorff method with $\chi(t)$ absolutely continuous on $[0,1]$. Then $V \cdot H \sim H$ for bounded sequences, where $V=$ the Cesàro method $C_{\alpha}$ $(\alpha>0)$ or the Abel method $A$ or the method $A \cdot C_{\alpha}(\alpha>-1)$.

Proof. Since $V$ is conservative, it follows that $H s \in(c)$ implies $V$ sums $H s$, i.e. $V \cdot H$ sums $s$. Now let $s \in(m)$ [the space of bounded sequences] and let $t=H s$ be defined by (1). We have to prove that

$$
s \in(m), V \text { sums } H s \text { imply that } H s \in(c) .
$$

Received by the editors July 10, 1986.

1980 Mathematics Subject Classification (1985 Revision). Primary 40G05, 40D20.

The work presented here was supported in part by a grant from the NSERC of Canada. 
Since slow oscillation is a Tauberian condition for $V$ [6, Theorem and Remarks 1-3] it is enough to prove that

$$
t_{n}-t_{m} \rightarrow 0 \text { as } n>m \rightarrow \infty, n / m \rightarrow 1 .
$$

In what follows, we take $n>m$ throughout and limits are always to be as $m \rightarrow \infty$, $n / m \rightarrow 1$. We write for $n, k \geqslant 0$ and $0 \leqslant t \leqslant 1$ :

$$
f_{n k}=\left(\begin{array}{l}
n \\
k
\end{array}\right) t^{k}(1-t)^{n-k}, \quad F_{n}(t)=\sum_{k=0}^{n} f_{n k}(t) s_{k} .
$$

Since $\chi(t)$ is absolutely continuous, it is an indefinite integral of a function $g(t)$, say. We have then

$$
t_{n}=\int_{0}^{1} F_{n}(t) g(t) d t
$$

Making an obvious change of variable in the equation obtained by replacing $n$ by $m$ in (4), we have

$$
\begin{aligned}
t_{n}-t_{m} & =\int_{0}^{1} F_{n}(t) g(t) d t-\frac{n}{m} \int_{0}^{m / n} F_{m}\left(\frac{n}{m} t\right) g\left(\frac{n}{m} t\right) d t \\
& =\int_{0}^{1}\left\{F_{n}(t) g(t)-\frac{n}{m} F_{m}\left(\frac{n}{m} t\right) g\left(\frac{n}{m} t\right)\right\} d t,
\end{aligned}
$$

where we take $g(u)=0$ for $u>1$. Thus

$$
\begin{aligned}
t_{n}-t_{m}= & \int_{0}^{1} F_{n}(t)\left\{g(t)-g\left(\frac{n}{m} t\right)\right\} d t \\
& +\left(1-\frac{n}{m}\right) \int_{0}^{1} F_{n}(t) g\left(\frac{n}{m} t\right) d t \\
& +\frac{n}{m} \int_{0}^{1} g\left(\frac{n}{m} t\right)\left[F_{n}(t)-F_{m}\left(\frac{n}{m} t\right)\right] d t \\
= & I_{1}+I_{2}+I_{3}, \text { say. }
\end{aligned}
$$

Since $\left|F_{n}(t)\right| \leqslant \sup \left|s_{k}\right|<\infty$

$$
I_{1}=O\left\{\int_{0}^{1}\left|g(t)-g\left(\frac{n}{m} t\right)\right| d t\right\} \rightarrow 0 .
$$

Also

$$
\int_{0}^{1} F_{n}(t) g\left(\frac{n}{m} t\right) d t=O(1)
$$

and hence $I_{2} \rightarrow 0$. In order to prove (3) it is therefore enough to prove that $I_{3} \rightarrow 0$.

Now since the expression inside the square brackets in the integral for $I_{3}$ is bounded, the contributions to $I_{3}$ of the range $(0, \eta)$ and $(1-\eta, 1)$ can be made arbitrarily small by choice of $\eta$. Thus it is enough to prove that for fixed $\eta$ with $0<\eta<\frac{1}{2}$ we have, uniformly in $\eta \leqslant t \leqslant 1-\eta$,

$$
F_{n}(t)-F_{m}\left(\frac{n}{m} t\right) \rightarrow 0 .
$$

Since $\left\{s_{k}\right\}$ is bounded, (5) will follow if we show that, uniformly in $\eta \leqslant t \leqslant 1-\eta$,

$$
\sum_{k=m+1}^{n} f_{n k}(t)+\sum_{k=0}^{m}\left|f_{n k}(t)-f_{m k}\left(\frac{n}{m} t\right)\right| \rightarrow 0 \text {. }
$$


We now appeal to [2, Theorem 138] (with the " $q$ " of that theorem taken as $(1-t) / t)$. Note that while [2] gives the result for fixed $t$, it does in fact hold uniformly in $\eta \leqslant t \leqslant 1-\eta$.

By clause (4) of that theorem, given $\varepsilon>0$ there is a number $\lambda$ such that for sufficiently large $n$,

$$
\sum_{1} f_{n k}(t)<\varepsilon
$$

where $\Sigma_{1}$ denotes summation over those $k$ for which $|k-n t|>\lambda n^{1 / 2}$. Since $t \leqslant 1-\eta$, the range of summation in (7) includes all $k \geqslant n(1-\eta)+\lambda n^{1 / 2}$, and, since $n / m \rightarrow 1$, it will ultimately include the whole range of the first sum in (6). Also, if $|k-n t|>\lambda n^{1 / 2}$, then

$$
\left|k-m \cdot \frac{n}{m} t\right|>\lambda n^{1 / 2}>\lambda m^{1 / 2},
$$

so that the following inequality similar to (7) holds also:

$$
\sum_{1} f_{m k}\left(\frac{n}{m} t\right)<\varepsilon
$$

Thus we need only show that for fixed $\lambda$,

$$
\sum^{*}\left|f_{n k}(t)-f_{m k}\left(\frac{n}{m} t\right)\right| \rightarrow 0
$$

(where $\Sigma^{*}$ denotes summation over those $k$ for which $|k-n t| \leqslant \lambda n^{1 / 2}$ ), uniformly in $[\eta, 1-\eta]$. In this range of values for $k$, clause (5) of [2, Theorem 138] is applicable, so that, uniformly in the relevant range of values for $k$,

$$
f_{n k}(t)=[2 \pi n t(1-t)]^{-1 / 2} A(n, k, t)\left[1+O\left(n^{-1 / 2}\right)\right],
$$

where

$$
A(n, k, t)=\exp \left\{\frac{-(k-n t)^{2}}{2 n t(1-t)}\right\}
$$

Also, replacing $n$ by $m$ and $t$ by $(n / m) t$, we get

$$
f_{m k}\left(\frac{n}{m} t\right)=\left[2 \pi n t\left(1-\frac{n}{m} t\right)\right]^{-1 / 2} A\left(m, k, \frac{n}{m} t\right)\left[1+O\left(n^{-1 / 2}\right)\right] .
$$

Since

$$
\sum[2 \pi n t(1-t)]^{-1 / 2} A(n, k, t)=O(1),
$$

the contribution to (8) of the " $O$ "-term in (9) is $O\left(n^{-1 / 2}\right)$. Similarly the contribution to $(8)$ of the " $O$ "-term in (10) is $O\left(n^{-1 / 2}\right)$. Thus, omitting the factor $(2 \pi t)^{-1 / 2}$ (as we may, since this is bounded), it is enough to prove that

$$
n^{-1 / 2} \sum^{*}\left|(1-t)^{-1 / 2} A(n, k, t)-\left(1-\frac{n}{m} t\right)^{-1 / 2} A\left(m, k, \frac{t}{m}\right)\right| \rightarrow 0,
$$


where the symbol $\Sigma^{*}$ denotes summation over the values of $k$ for which $|k-n t| \leqslant$ $\lambda n^{1 / 2}$. The expression on the left-hand side of (12) is less than or equal to

$$
\begin{aligned}
& n^{-1 / 2} \sum^{*}\left|(1-t)^{-1 / 2}-\left(1-\frac{n}{m} t\right)^{-1 / 2}\right| A(n, k, t) \\
& \quad+n^{-1 / 2} \sum^{*}\left(1-\frac{n}{m} t\right)^{-1 / 2}\left|A(n, k, t)-A\left(m, k, \frac{n}{m} t\right)\right| \\
& =J_{1}+J_{2}, \quad \text { say. }
\end{aligned}
$$

Thus, in order to prove (8) and the Theorem, it is enough to prove that $J_{1}$ and $J_{2}$ tend to 0 uniformly. Now since the function $h(t)=(1-t)^{-1 / 2}$ has a bounded derivative in the interval $\eta \leqslant t \leqslant 1-\eta$, the expression inside the modulus in $J_{1}$ is

$$
h(t)-h\left(\frac{n}{m} t\right)=O\left(\left|1-\frac{n}{m}\right| t\right)=o(1) .
$$

It follows from (11) that $J_{1} \rightarrow 0$.

Now, if $0<p<q$, then

$$
0<e^{-p}-e^{-q}=\int_{p}^{q} e^{-x} d x<(q-p) e^{-p} .
$$

Hence the expression inside the modulus in $J_{2}$ is positive and less than

$$
\begin{gathered}
(k-n t)^{2}\left[\frac{1}{2 n t(1-(n / m) t)}-\frac{1}{2 n t(1-t)}\right] A(n, k, t) \\
=(k-n t)^{2} \frac{(n / m)-1}{2 n(1-(n / m) t)(1-t)} A(n, k, t) .
\end{gathered}
$$

Since $n / m-1=o(1)$ and the function $(1-t)^{-1}$ and $(1-(n / m) t)^{-1}$ are bounded, it follows that

$$
J_{2}=o\left\{n^{-3 / 2} \sum^{*}(k-n t)^{2} A(n, k, t)\right\} .
$$

But the expression in curly brackets is bounded. Hence $J_{2} \rightarrow 0$, and the proof of the Theorem is complete.

RemarKs. (1) The Theorem gives a sufficient condition in order that a conservative Hausdorff method $H=(H, \chi)$ will be equivalent to $V \cdot H$ for bounded sequences. But the absolute continuity of $\chi$ is not necessary for this equivalence, as is shown by the following example: For an arbitrary number $\lambda$, let $K_{\lambda}=(H, g)$ be the Hausdorff matrix generated by the function $g$ with $g(0)=0$ and $g(t)=\lambda$ for $0<t \leqslant 1$. Then $K_{\lambda}$ sums every bounded sequence $\left\{s_{n}\right\}$ to $\lambda s_{0}$, and hence for any Hausdorff method $H=(H, \chi)$ with $\chi \in \mathrm{AC}[0,1], H+K_{\lambda} \sim C_{1}\left(H+K_{\lambda}\right)$. But the generating function $\chi+g$ of $H+K_{\lambda} \notin \mathrm{AC}[0,1]$ if $\lambda \neq 0$.

(2) Subsequent to the completion of this paper we have succeeded in proving that a conservative Hausdorff method $P$ will be equivalent to $V \cdot P$ for bounded sequences if and only if $P$ is of the form $H+K_{\lambda}$, where $H=(H, \chi)$ with $\chi \in \mathrm{AC}[0,1]$. In particular, if $H=(H, \chi)$ is a multiplicative Hausdorff method, then $H \sim V \cdot H$ for bounded sequences if and only if $\chi \in A C[0,1]$. Thus the Theorem is a best possible one for multiplicative Hausdorff methods in the sense that the condition $\chi \in A C[0,1]$ cannot be weakened. 
(3) The Theorem is also best possible in the sense that it does not hold for sequences bounded on only one side. To see this, it is enough to consider the case $V=C_{\alpha}(0<\alpha<1)$, since of $0<\alpha<\beta, C_{\alpha} H \subset C_{\beta} H \subset A \cdot H$. Take $H=C_{1-\alpha}$ and a sequence $s$ such that $s_{n} \geqslant 0, C_{1} s \in(c)$ [and hence $C_{\alpha} \cdot C_{1-\alpha} s \in(c)$ ] but $C_{1-\alpha} \notin(c)$ (e.g. let $s_{n}=2^{(1-\alpha) k}$ if $n=2^{k}$ and $s_{n}=0$ otherwise, for $n, k=$ $0,1, \ldots)$.

(4) The proof of the Theorem shows that the result is true for an arbitrary conservative method $V$ for which slow oscillation is a Tauberian condition. It is therefore true for any conservative method $V$ which has $n a_{n}=O(1)$ as a Tauberian condition and for which $V \subset V \cdot C_{1}$. (To see this, adapt the theorem and proof given in [6].) The methods $V$ considered in the Theorem of the present paper are all of this type.

(5) If $A, B$ are matrices, let us write $A \approx B$ if $(A-B) x \in(c)$ for every $x \in(m)$ (see [8, p. 69] on "volläquivalenz"). Our Theorem says that if $\chi \in \mathrm{AC}[0,1]$ and $H=(H, \chi)$, then $H \sim H C_{1}$ for bounded sequences; however we cannot assert that

$$
H \approx H C_{1} \quad \text { i.e. }\left(H-H C_{1}\right) x \in(c) \text { for every } x \in(m) .
$$

For suppose that (13) holds when $H=C_{1}$. ( $C_{1}$ has generating function $\chi(t)=t \in$ $\mathrm{AC}[0,1]$.) Since $C_{1}-C_{1} C_{1}$ is multiplicative, it follows (by [2, Theorem 3]) that, for every $x \in(m)$,

$$
C_{1}\left(I-C_{1}\right) x=\left(C_{1}-C_{1} C_{1}\right) x \in\left(c_{0}\right)
$$

and hence

$$
C_{2}\left(I-C_{1}\right) x=\left(C_{2}-C_{2} C_{1}\right) x \in\left(c_{0}\right) .
$$

We have then both the relations

$$
C_{1} C_{1} \approx C_{1} \text { and } C_{1} C_{2} \approx C_{2} \text {. }
$$

Since $C_{1} C_{1}-C_{2}=\left(C_{1} C_{2}-C_{2}\right) / 2$ (see [2, p. 107 or 8, p. 108]), it follows from (16) that $C_{1} C_{1} \approx C_{2}$ and hence $C_{1} \approx C_{1} C_{2} \approx C_{2}$. Since $C_{1}-C_{2}$ is multiplicative and $C_{1} \approx C_{2}$, we must have then

$$
\left(C_{1}-C_{2}\right) x \in\left(c_{0}\right) \text { for every bounded sequence } x \text {. }
$$

But Cooke [1, pp. 118-119] has proved that (17) is not true. Hence (13) is false when $H=C_{1}$.

\section{REFERENCES}

1. R. G. Cooke, On mutual consistency and regular T-limits, Proc. London Math. Soc. (2) 41 (1936), 113-125.

2. G. H. Hardy, Divergent series, Oxford Univ. Press, Oxford, 1949.

3. B. Kuttner and M. R. Parameswaran, A product theorem and a Tauberian theorem for Euler methods, J. London Math. Soc. (2) 18 (1978), 299-304.

4. M. R. Parameswaran, On a generalization of a theorem of Meyer-König, Math. Z. 162 (1978), 201-204.

5. __ A converse product theorem in summability, Comment. Math. 22 (1980), 131-134. 
6. __ A general Tauberian theorem, Glasnik Mat. 14 (34) (1979), 83-86.

7. A. J. Stam, Wiener's Tauberian theorem for Hausdorff limitation methods, Nieuw Arch. Wisk. (3) 25 (1977), 182-185.

8. K. Zeller and W. Beekmann, Theorie der Limitierungsverfahren, Springer-Verlag, Berlin and New York, 1970.

Department of Pure Mathematics, The University, Birmingham, B15 2TT, United Kingdom

Department of Mathematics \& Astronomy, University of Manitoba, Winnipeg, Manitoba R3T 2N2, CANADA 